\title{
Implementação de método adaptativo no tempo para equações diferenciais parciais evolutivas
}

\author{
Muller M. S. Lopes \\ Curso de Pós Graduação em Computação Aplicada, CTE/INPE, \\ 12227-010, São José dos Campos, SP \\ E-mail:mullermslopes@gmail.com \\ Margarete O. Domingues \\ Laboratório Associado de Computação e Matemática Aplicada, CTE/INPE, \\ 12227-010, São José dos Campos, SP \\ E-mail: margarete@lac.inpe.br \\ Odim Mendes \\ Divisão de Geofísica Espacial, CEA/INPE \\ 12227-010, São José dos Campos, SP \\ E-mail:odim.mendes@inpe.br
}

\section{RESUMO}

Resoluções numéricas de Equações Diferenciais Parciais (EDPs) evolutivas com soluções que apresentam poucas estruturas localizadas podem ter um alto custo computacional. Tornam-se assim importantes estudos realizados que visem desenvolver técnicas que reduzam este custo. Uma dessas técnicas é a de análise multirresolução no contexto de volumes finitos (VF) proposta por Ami Harten [2]. Nessa técnica, malhas adaptadas à solução podem ser construídas com base na avaliação da regularidade local da solução a cada instante de tempo. Uma malha menos refinada é utilizada nas regiões que apresentam maior regularidade. Uma adaptabilidade no tempo também é importante para uma maior aproveitamento da redução de custos dessas malhas adaptativas. Em [1], uma técnica de tempo local foi desenvolvida. Neste trabalho, que faz parte da Dissertação de Mestrado [3], estende-se essa ideia para ordens superiores utilizando outra metodologia de evolução temporal.

Os resultados obtidos com a técnica de tempo local proposta (MR/LT/RK3) de ordem três são comparados com a técnica sem as estratégias de tempo local. Mais detalhes dessas técnicas para ordem dois são apresentados em [1]. Nesse caso, as técnicas sem estratégia de tempo local são realizadas com métodos explícitos de Runge-Kutta de ordem 3 ( denominado como MR/RK3). É utilizado o método VF tradicional no nível mais fino de refinamento para avaliar o erro cometido na solução.

O ganho em relação ao método sem adaptabilidade temporal é calculado como gain = $1-\frac{\text { erro }_{M R / L T}}{\operatorname{erro} o_{M R}} \cdot \frac{\text { eempo }_{M R / L T}}{\text { tempo }_{M R}}$. Os valores erro e tempo são respectivamente o erro médio e o tempo de CPU dos métodos considerados. O valor gain indica, em \%, o ganho do método proposto em relação ao MR.

Este estudo apresenta a eficiência deste método na resolução numérica da Equação de Burgers, que representa um modelo simples de turbulência, e é dada por:

$$
\frac{\partial U(x, t)}{\partial t}+\frac{\partial\left(\frac{1}{2} U^{2}(x, t)\right)}{\partial x}=\mu \frac{\partial^{2} U(x, t)}{\partial x^{2}},
$$

em que $t>0$ e $\mu=0,003183099$ é o coeficiente de viscosidade. 
Para o caso unidimensional, este modelo é resolvido numericamente utilizando as seguintes condições iniciais e de contorno: $U(0, t)=U(1, t)=0, U(x, 0)=\sin (2 \pi x)$.

Para este caso, a simulação é realizada até o instante 0,4 , utilizando a constante CFL: $\sigma=0,5$ e um valor de threshold $\epsilon=10^{-3}$. Estas simulações foram realizadas utilizando um computador com processador Intel (R) Atom CPU 1, 6GHz.

Os resultados comparativos de tempo computacional, memória utilizada e erro médio obtido são apresentados na Tabela. Esses resultados são obtidos para malhas com diversos de níveis de refinamento. Para o melhor caso, $L=13$, o método proposto apresentou um valor de gain de 44\%. O método se mostrou mais eficiente a medida em que são utilizadas malhas com mais níveis de refinamento Conforme apresentado na Tabela, o método proposto apresentou o mesmo custo em memória e um menor tempo computacional em relação ao método MR/RK3, conquanto não houve alteração na ordem do erro obtido.

\begin{tabular}{|c|c|c|c|c|}
\hline & \multirow[t]{2}{*}{ Método } & \multirow{2}{*}{$\begin{array}{l}\text { Erro médio } \\
\left(\times 10^{-1}\right)\end{array}$} & \multicolumn{2}{|c|}{ CPU (\%) } \\
\hline & & & Tempo & Memória \\
\hline \multirow{3}{*}{$L=11$} & $\mathrm{VF} / \mathrm{RK} 3$ & & 100 & 100 \\
\hline & MR/RK3 & 0,56 & 35 & 23 \\
\hline & MR/LT/RK3 & 0,68 & 30 & 23 \\
\hline \multirow{3}{*}{$L=12$} & VF/RK3 & & 100 & 100 \\
\hline & MR/RK3 & 1,00 & 18 & 12 \\
\hline & MR/LT/RK3 & 1,10 & 12 & 12 \\
\hline \multirow{3}{*}{$L=13$} & VF/RK3 & & 100 & 100 \\
\hline & $\mathrm{MR} / \mathrm{RK} 3$ & 1,50 & 10 & 6 \\
\hline & MR/LT/RK3 & 1,70 & 5 & 6 \\
\hline
\end{tabular}

Tabela 1: Comparativo dos erros, tempos de processamento e economia de memória obtidas utilizando os métodos VF/RK3, MR/RK3 e a técnica MR/LT/RK3 proposta. $\epsilon=10^{-3}$. Tempo final: $t_{f}=0,4$.

Nota: Tempos computacionais obtidos pelo método VF/RK3: 6, 4 min $(L=11) ; 53,1$ min $(L=12) ; 398,8$ min $(L=13)$.

\section{Referências}

[1] DOMingueS, M. O.; GOMES, S. M.; ROUSSEL, O.; SCHNEIDER, K. An adaptive multiresolution scheme with local time stepping for evolutionary PDEs. J. Comput. Phys., v. 227 , n. 8 , p. $3758-3780$, abr. 2008.

[2] HARTEN, A. Adaptive multiresolution schemes for shock computations. J. Comput. Phys., v. 115 , p. $319-338,1994$. ISSN 1270-900X/e.

[3] LOPES, M. ,Método de alta ordem para ajuste de passo de tempo local para resolução numérica de equações diferenciais evolutivas com uso de análise multirresolução adaptativa. Dissertação de Mestrado - Instituto Nacional de Pesquisas Espaciais, 2014. 